\title{
Mediterranean diet and cancer
}

\author{
Carlo La Vecchia ${ }^{1,2, *}$ \\ 'Istituto di Ricerche Farmacologiche 'Mario Negri', via Eritrea 62, 20157 Milano, Italy: ${ }^{2}$ Istituto di Statistica \\ Medica e Biometria, Università di Milano, Milano, Italy
}

\begin{abstract}
Objective: To analyse the role of various aspects of the Mediterranean diet in several common epithelial cancers, including digestive and selected non-digestive tract neoplasms.

Design: Systematic analysis of data from a series of case-control studies.

Setting: Northern Italy, between 1983 and 1998.

Subjects: Over 12000 cases of 20 cancer sites and 10000 controls.

Results: For most epithelial cancers, the risk decreased with increasing vegetable and fruit consumption, with relative risk (RR) between 0.3 and 0.7 for the highest versus the lowest tertile. For digestive tract cancers, population-attributable risks for low intake of vegetables and fruit ranged between 15 and $40 \%$. A protective effect was observed also for breast, female genital tract, urinary tract and a few other epithelial neoplasms. A number of antioxidants and other micronutrients showed an inverse relationship with cancer risk, but the main components responsible for the favourable effect of a diet rich in vegetables and fruit remain undefined. Fish tended to be another favourable diet indicator. In contrast, subjects reporting frequent red meat intake showed RRs above unity for several common neoplasms. Intake of wholegrain foods was related to a reduced risk of several types of cancer, particularly of the upper digestive tract. This may be due to a favourable role of fibre, but the issue is still open to discussion. In contrast, refined grain intake and, consequently, glycaemic load and glycaemic index were associated with increased risk of different types of cancer including, among others, breast and colorectal.

Conclusions: A low-risk diet for cancer in the Mediterranean would imply increasing the consumption of fruit and vegetables, as well as avoiding increasing the intakes of meat and refined carbohydrates. Further, olive oil and other unsaturated fats, which are also typical aspects of the Mediterranean diet, should be preferred to saturated ones.
\end{abstract}

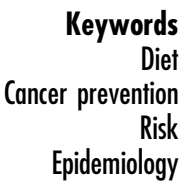

Several aspects of the Mediterranean diet have been related to a reduced risk not only of cardiovascular disease, but also of several cancers. It has been estimated that up to $25 \%$ of colorectal, $15 \%$ of breast and $10 \%$ of prostate, pancreas and endometrial cancers could be prevented by shifting to a healthy Mediterranean diet $^{1}$.

In the present paper, the main findings from an integrated series of case-control studies conducted in Italy are reviewed.

\section{Vegetables, fruit and micronutrients}

The relationship between the frequency of consumption of vegetables and fruit and cancer risk was analysed using data from a series of case-control studies conducted in Italy since 1983, and including data collected until 1998. Overall, these included more than 12000 cases of various cancer sites and 10000 controls. The design and methods of the various studies included in this systematic overview have been described elsewhere ${ }^{2-8}$. The relative risk (RR) for digestive tract neoplasms ranged from 0.3 to 0.7 for the highest versus the lowest tertile of vegetable intake (Fig. 1). Protective effects were also observed for several hormonerelated neoplasms. Fruit was related to a reduced RR for cancers of the upper digestive tract, stomach and the urinary tract (Fig. 2). For digestive tract cancers, population-attributable risk for low intake of vegetables and fruit ranged between 15 and $40 \%{ }^{2}$.

The role of selected micronutrients and antioxidants was also considered. $\beta$-Carotene, vitamin $\mathrm{E}$ and calcium showed an inverse relationship with breast cancer risk ${ }^{3}$. For colorectal cancer, the RR reached 0.46 in subjects reporting high calcium/vitamin D and high antioxidant intakes, compared with subjects reporting low intakes of both groups of micronutrients ${ }^{4}$. Still, the major nutrients or micronutrients that may be responsible for the favourable effect of fruit and vegetables remain undefined.

\section{Meat and fish}

Other aspects of diet are also likely to influence cancer risk, and among these, red meat is of specific relevance. 


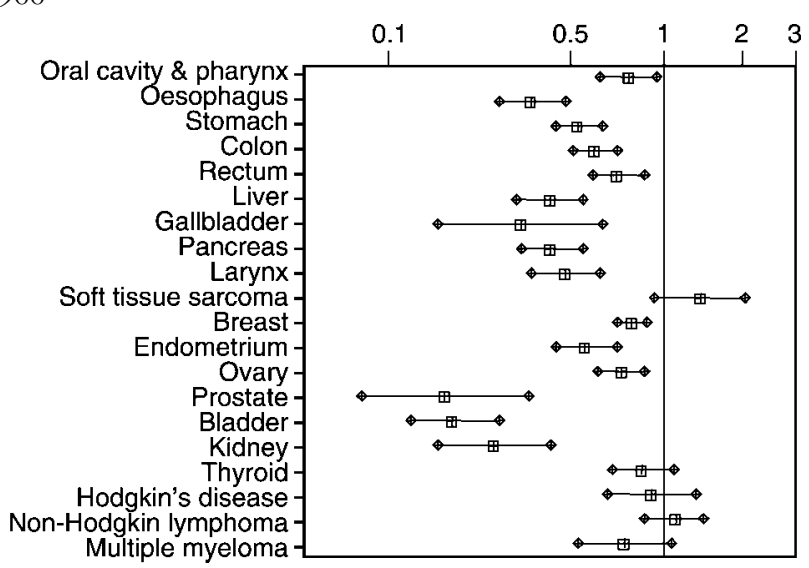

Fig. 1 Relative risk and 95\% confidence interval of selected cancers for the highest versus the lowest tertile of vegetable consumption. Italy, 1983-1998

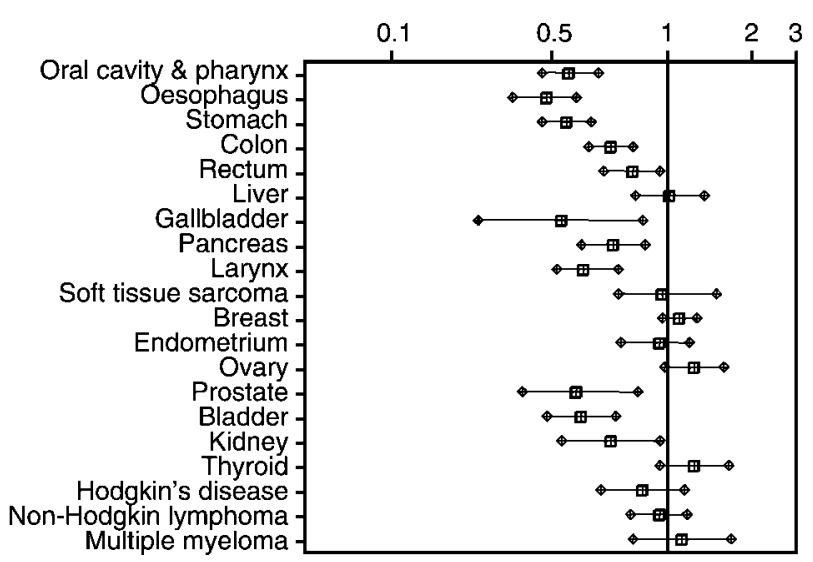

Fig. 2 Relative risk and 95\% confidence interval of selected cancers for the highest versus the lowest tertile of fruit consumption. Italy, 1983-1998

In an integrated series of studies from Italy, the multivariate RR for the highest tertile of meat intake $(\geq 7$ times per week) compared with the lowest ( $\leq 3$ times per week) was 1.7 for stomach, 2.0 for colon, 1.9 for rectal, 1.6 for pancreas, 1.5 for bladder, 1.5 for endometrial and 1.3 for ovarian cancers (Fig. 3). This points to meat intake as another important factor (after vegetables and fruits) in the

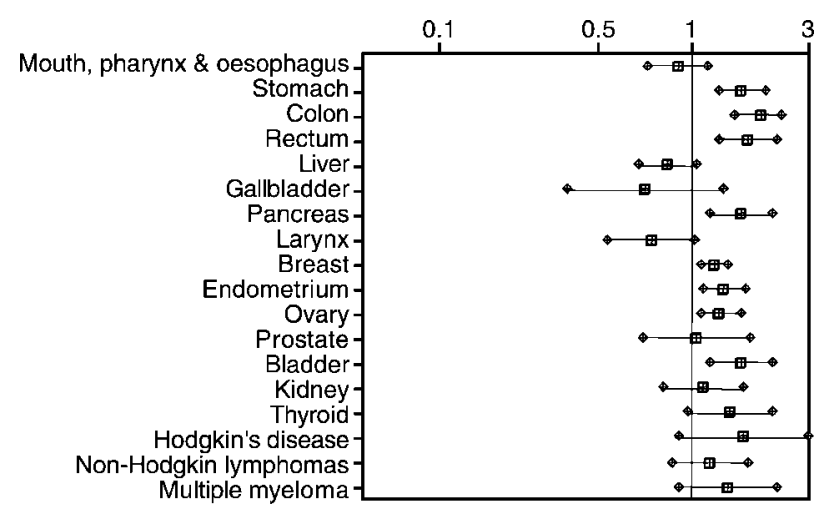

Fig. 3 Relative risk and 95\% confidence interval of selected cancers for the highest versus the lowest tertile of red meat consumption. Italy, 1983-1998 nutritional aetiology of human cancer ${ }^{5}$. This message is of particular importance for Mediterranean populations, whose traditional diet does not include a high intake of red meat, but who have been changing their dietary habits in an unfavourable way ${ }^{1}$.

In contrast, frequent fish intake appears to be a favourable indicator for reduced risk of several common cancers $^{6}$, mostly of the digestive tract but also of the endometrium, ovary and prostate (Fig. 4).

\section{Fibres, whole and refined cereals}

Intake of whole-grain foods has been related to reduced risk of colorectal cancer, as well as cancer of several other sites. In the Italian network of case-control studies, the RR for the highest consumption level of whole-grain foods was $0.2-0.3$ for upper digestive and respiratory tract neoplasms, 0.5 for stomach, colon and gallbladder cancers, 0.7 for rectal cancer, 0.6 for liver cancer, 0.8 for pancreas and prostate cancer, 0.9 for breast and endometrial cancers, 0.6 for ovarian cancer, 0.4 for bladder and kidney cancers, and about 0.5 for lymphomas and myelomas (Fig. 5). Thus, even in the absence of an unequivocal and satisfactory biological interpretation, in this Mediterranean population higher intake frequency of whole-grain foods is an indicator of reduced cancer risk ${ }^{7}$.

With reference to a specific role of fibres in colorectal carcinogenesis, we considered data from a case-control study conducted in Italy $^{8}$, an area with intermediate colorectal cancer incidence and mortality on a European level ${ }^{9}$. Cases were 1953 incident, histologically confirmed colorectal cancers (1225 of the colon and 728 of the rectum), admitted to the major teaching and general hospitals in the study areas, and controls, 4154 subjects admitted to hospitals in the same catchment areas for acute, non-neoplastic diseases. For most types of fibre, the RR of colon and rectal cancers was significantly below unity, and no appreciable differences emerged between the two sites. When the unit was set at the difference between the upper

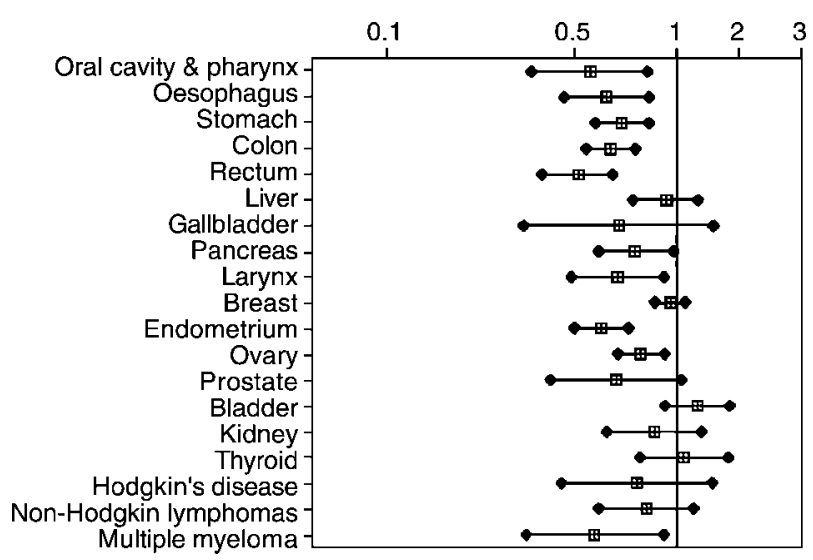

Fig. 4 Relative risk and 95\% confidence interval of selected cancers for the highest versus the lowest tertile of fish consumption. Italy, 1983-1998 


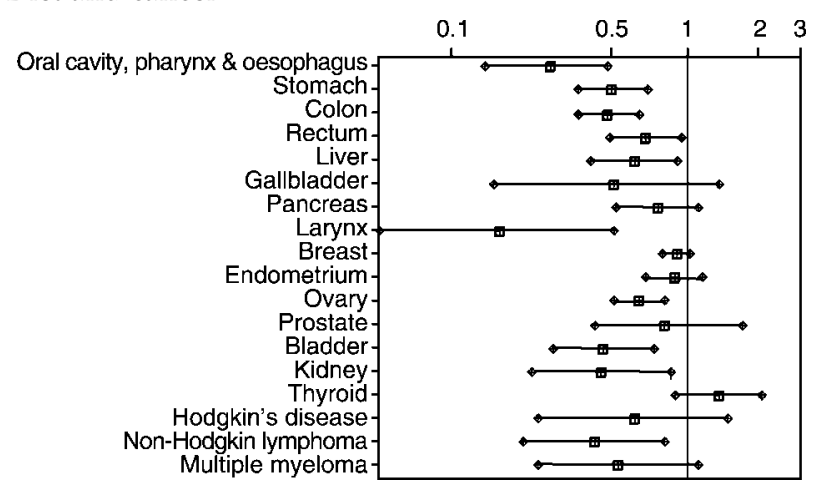

Fig. 5 Relative risk and $95 \%$ confidence interval of selected cancers for the highest versus the lowest tertile of whole-grain food consumption. Italy, 1983-1998

cut points of the 80th and 20th percentiles, the RR for colorectal cancer was 0.68 for total fibres, 0.67 for soluble non-cellulose polysaccharides (NCP), 0.71 for total insoluble fibre, 0.67 for cellulose, 0.82 for insoluble NCP and 0.88 for lignin. When fibre was classified according to the source, the RR was 0.75 for vegetable fibre and 0.85 for fruit fibre, but 1.09 for cereal fibre.

In contrast, intake of refined grains was associated with increased risk of cancers of the stomach, colon-rectum, breast, upper digestive tract sites and thyroid in studies conducted in Mediterranean populations ${ }^{10}$ (Fig. 6). Glycaemic load has consequently been suggested to be a relevant factor in colorectal and breast carcinogenesis, pointing to a potential role of insulin-like growth factors $^{11-13}$.

Whole-grain foods should therefore replace refined cereal ones, whenever possible.

\section{Fats}

The issue of fats, and of specific types of fat, on the risk of colorectal and breast cancers, as well as of several other neoplasms, remains a major open issue on a prevention and public health level. In large studies from Italy, isocaloric substitution of $5 \%$ of total calories as saturated fats by unsaturated ones was associated with reductions in breast (odds ratio, $\mathrm{OR}=0.67)$ and colorectal $(\mathrm{OR}=0.78)$ cancer risk. Replacement of complex carbohydrates by unsaturated fats was associated with similar risk

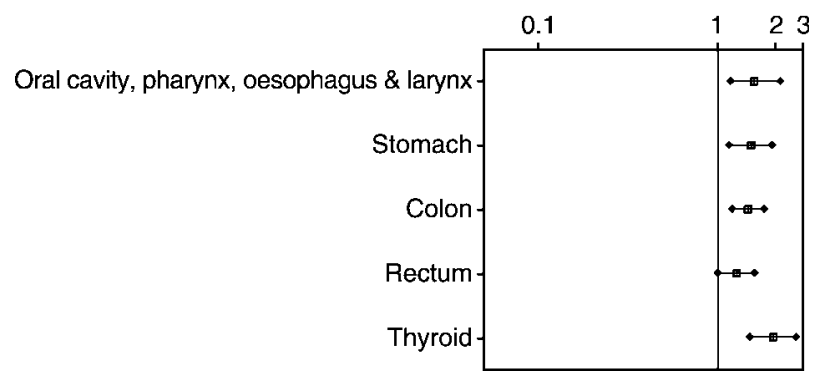

Fig. 6 Relative risk and 95\% confidence interval of selected cancers for the highest versus the lowest level of refined cereal consumption. Italy, 1983-1998 reductions. Part of the benefit for mono- and polyunsaturated fats in the Mediterranean diet may be due to the positive correlation between (olive) oil and vegetable intake $^{10}$.

It seems therefore that substituting olive oil for other seasoning fats has favourable effects on the risk of breast $^{10,14-16}$ and colorectal ${ }^{17}$ cancers, as well as upper digestive tract neoplasms, with the highest consumption level of olive oil giving RR of 0.7 for oral and pharyngeal cancer $^{18}$ and RR of 0.4 for oesophageal cancer ${ }^{19}$.

\section{Conclusions}

In conclusion, progress has been made in our understanding of dietary correlates of the process of carcinogenesis, but a few aspects (e.g. refined cereals) have come under a process of critical re-evaluation ${ }^{20,21}$.

In Mediterranean populations, in any case, a low-risk diet for cancer would imply not only increasing fruit and vegetable consumption ${ }^{22}$, but also avoiding increasing red meat as well as refined carbohydrate intakes, and preferring olive oil and other unsaturated fats to saturated ones ${ }^{16,23}$.

\section{Acknowledgements}

This work was conducted with the contribution of the Italian Association for Cancer Research and the Italian League against Cancer. Ms Ivana Garimoldi provided editorial assistance.

\section{References}

1 Trichopoulou A, Lagiou P, Kuper H, Trichopoulos D. Cancer and Mediterranean dietary traditions. Cancer Epidemiology, Biomarkers \& Prevention 2000; 9: 869-73.

2 La Vecchia C, Chatenoud L, Franceschi S, Soler M, Parazzini F, Negri E. Vegetables and fruit and human cancer: update of an Italian study. International Journal of Cancer 1999; 82: $151-2$.

3 Negri E, La Vecchia C, Franceschi S, D’Avanzo B, Talamini R, Parpinel $\mathrm{M}$, et al. Intake of selected micronutrients and the risk of breast cancer. International Journal of Cancer 1996; 65: $140-4$.

4 La Vecchia C, Braga C, Negri E, Franceschi S, Russo A, Conti $\mathrm{E}$, et al. Intake of selected micronutrients and the risk of colorectal cancer. International Journal of Cancer 1997; 73 : 525-30.

5 Tavani A, La Vecchia C, Gallus S, Lagiou P, Trichopoulos D, Levi F, et al. Red meat intake and cancer risk: a study in Italy. International Journal of Cancer 2000; 86: 425-8.

6 Fernandez E, Chatenoud L, La Vecchia C, Negri E, Franceschi S. Fish consumption and cancer risk. American Journal of Clinical Nutrition 1999; 70: 85-90.

7 Chatenoud L, Tavani A, La Vecchia C, Jacobs DR Jr, Negri E, Levi $\mathrm{F}$, et al. Whole grain food intake and cancer risk. International Journal of Cancer 1998; 77: 24-8.

8 Negri E, Franceschi S, Parpinel M, La Vecchia C. Fibre intake and risk of colorectal cancer. Cancer Epidemiology, Biomarkers \& Prevention 1998; 7: 667-71.

9 Levi F, Lucchini F, Boyle P, Negri E, La Vecchia C. Cancer 
incidence and mortality in Europe, 1988-92. Journal of Epidemiology and Biostatistics 1998; 3: 295-361.

10 Franceschi S, Russo A, La Vecchia C. Carbohydrates, fat and cancer of the breast and colon-rectum. Journal of Epidemiology and Biostatistics 1998; 3: 217-8.

11 Giovannucci E. Insulin and colon cancer. Cancer Causes $\mathcal{E}$ Control 1995; 6: 164-79.

12 Franceschi S, Dal Maso L, Augustin L, Negri E, Parpinel M, Boyle P, et al. Dietary glycemic load and colorectal cancer risk. Annals of Oncology 2001; 12: 173-8.

13 Augustin LS, Dal Maso L, La Vecchia C, Parpinel M, Negri E, Vaccarella S, et al. Dietary glycemic index and glycemic load, and breast cancer risk: a case-control study. Annals of Oncology 2001; 12: 1533-8.

14 La Vecchia C, Negri E, Franceschi S, Decarli A, Giacosa A, Lipworth L. Olive oil, other dietary fats, and the risk of breast cancer (Italy). Cancer Causes \& Control 1995; 6: 545-50.

15 Prieto-Ramos F, Serra-Majem L, La Vecchia C, Ramon JM, Tresserras R, Salieras L. Mortality trends and past and current dietary factors of breast cancer in Spain. European Journal of Epidemiology 1996; 12: 141-8.

16 Trichopoulou A. Olive oil and breast cancer. Cancer Causes E Control 1995; 6: 545-50.

17 Braga C, La Vecchia C, Franceschi S, Negri E, Parpinel M,
Decarli A, et al. Olive oil, other seasoning fats, and the risk of colorectal carcinoma. Cancer 1998; 82: 448-53.

18 Franceschi S, Favero A, Conti E, Talamini R, Volpe R, Negri E, et al. Food groups, oils and butter, and cancer of the oral cavity and pharynx. British Journal of Cancer 1999; 80: 614-20.

19 Bosetti C, La Vecchia C, Talamini R, Simonato L, Zambon P, Negri E, et al. Food groups and risk of squamous cell esophageal cancer in Northern Italy. International Journal of Cancer 2000; 87: 289-94.

20 Willett WC. Diet and cancer: one view at the start of the millennium. Cancer Epidemiology, Biomarkers \& Prevention 2001; 10: 3-8.

21 Terry P, Giovannucci E, Michels KB, Bergkvist L, Hansen H, Holmberg L, et al. Fruit, vegetables, dietary fiber, and risk of colorectal cancer. Journal of the National Cancer Institute 2001; 93: 525-33.

22 La Vecchia C. Nutrizione e Tumori. Implicazioni di Prevenzione e Prospettive di Ricerca. Rome: Il Pensiero Scientifico Editore, 1997.

23 La Vecchia C, Chatenoud L, Altieri A, Tavani A. Nutrition and health: epidemiology of diet, cancer and cardiovascular disease in Italy. Nutrition, Metabolism, and Cardiovascular Diseases 2001; 11(Suppl. 4): 10-5. 